\title{
Defect density and recombination lifetime in microcrystalline silicon absorbers of highly efficient thin-film solar cells determined by numerical device simulations
}

\author{
T. Brammera) and H. Stiebig \\ Institute of Photovoltaics, Forschungszentrum Jülich GmbH, D-52425 Jülich, Germany
}

(Received 21 October 2002; accepted 9 April 2003)

\begin{abstract}
The absorber layers of microcrystalline silicon thin-film solar cells with $p-i-n$ structure deposited by plasma-enhanced chemical vapor deposition at $200{ }^{\circ} \mathrm{C}$ are characterized regarding the defect density and the recombination lifetime. The characterization is based on a comparison of experimentally determined solar cell characteristics with results from numerical device simulations. Evaluation of the dark reverse saturation current indicates a strong dependence of the recombination lifetime $\tau$ on the hydrogen dilution during the deposition. Close to the transition region to amorphous growth, where the highest solar cell efficiencies are observed, $\tau$ is maximum within the crystalline deposition regime and equals around $80 \mathrm{~ns}$. The aspect of a spatially varying defect density within the absorber layer is also addressed by numerical simulations. The results from the analysis of the dark current are compared with electron spin resonance data determined on single layers, which allows conclusions to be drawn regarding the capture cross section of the dominant recombination site in microcrystalline silicon. (C) 2003 American Institute of Physics. [DOI: 10.1063/1.1577813]
\end{abstract}

\section{INTRODUCTION}

The preferred deposition techniques for thin-film microcrystalline silicon $(\mu c-\mathrm{Si}: \mathrm{H})$ are plasma-enhanced chemical vapor deposition (PECVD) and hot-wire chemical vapor deposition. Both approaches have proven to yield solar cell efficiencies of about $9 \%$ in single junction cells ${ }^{1,2}$ and above $10 \%$ in combination with an amorphous solar cell forming a tandem solar cell. ${ }^{3-5}$ The process gases for CVD are silane $\left(\mathrm{SiH}_{4}\right)$ and hydrogen $\left(\mathrm{H}_{2}\right)$. The ratio of the two gas fluxes termed silane concentration $\mathrm{SC}=\left[\mathrm{SiH}_{4}\right] /\left(\left[\mathrm{SiH}_{4}\right]+\left[\mathrm{H}_{2}\right]\right)$ is crucial for the microstructure of the deposited silicon film. Previous investigations showed that films deposited at 95 $\mathrm{MHz}$ (VHF) are highly crystalline but porous when deposited at small SC $(<3 \%)$. At medium SC, below the transition region to amorphous growth $(3 \%<\mathrm{SC}<6 \%)$, the films are more compact, and for a high value of SC $(>6 \%)$, amorphous growth conditions prevail. ${ }^{6}$ Note that the dependence of the microstructure on the SC value varies with the deposition conditions (substrate material, deposition pressure, VHF power, frequency, and substrate temperature) so that the values for SC mentioned here might deviate for other deposition conditions. The highest solar cell efficiencies are achieved when the absorber layer ( $i$ layer) is deposited in the medium SC range, close to the transition region to amorphous growth. ${ }^{7}$ This is an indication that the electronic material properties also vary considerably with SC. However, measurements of electronic material properties such as the mobility-lifetime product $\mu \tau$ or the Hall mobility face many problems, since straightforward material characterization methods are not applicable due to the anisotropic (columnar growth), heterogeneous (crystalline and amorphous phase,

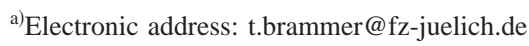

grain boundaries), and partly porous structure of $\mu c-\mathrm{Si}: \mathrm{H}$ films. Most of the conventional characterization techniques (Hall effect, photo/dark conductivity) analyze the transport properties perpendicular to the growth direction of the layers. In solar cells, however, the relevant direction regarding transport and recombination is the growth direction. Additionally, the growth and, consequently, the material properties depend strongly on the substrate material, ${ }^{8}$ so that material properties determined on single films deposited on, e.g., glass or metal, do not necessarily match the properties of films implemented in devices. A process usually neglected in work on $\mu c-\mathrm{Si}: \mathrm{H}$ is the recombination of charge carriers at the silicon/air interface via unsaturated surface states. This would strongly affect the mobility-lifetime product as measured by all photoconductivity experiments. Finally, there are no reports on recombination lifetimes or capture cross sections of defects in $\mu c$-Si:H as implemented in devices.

To summarize the current knowledge on $\mu c-\mathrm{Si}: \mathrm{H}$, a quantitative model to correlate electronic material properties and solar cell performance has not been established. Some explanations have been proposed. ${ }^{9,10}$ The reason why the highest solar cell efficiencies are achieved close to the transition region to amorphous growth is of particular interest. Therefore, it is the goal of this work to determine material properties from a comparison of experimentally determined diode properties with the results of numerical device simulations. In other words, we use numerical device simulations as an analysis tool to extract quantitative values for the electronic material properties, namely, the defect density and the recombination lifetime, of $\mu c-\mathrm{Si}: \mathrm{H}$ absorber layers. In that way, the intention of this work differs from that of other simulation studies on $\mu c-\mathrm{Si}: \mathrm{H}$, in which general aspects, e.g., doping of the absorber layer, ${ }^{11,12}$ the grain size, ${ }^{12,13}$ the band gap discontinuities at the grain boundaries, ${ }^{14,15}$ and an 
$a$-Si:H layer in front of the $\mu c$-Si:H $p$ layer, ${ }^{16}$ were investigated. Taretto et al. also compared the results from simulations with experimental data in order to determine the grain boundary defect density. ${ }^{12}$ These simulations were restricted, for mathematical reasons, to grain sizes of $1 \mu \mathrm{m}$. However, the diameter of the columnar grains in $\mu c-\mathrm{Si}: \mathrm{H}$ films is typically smaller than $100 \mathrm{~nm} \cdot{ }^{17}$

The structure of this paper is as follows. First, the dark $I / V$ curves as a function of SC are presented. After introducing the main features of our device simulation program, we discuss the effect of the defect density in the $i$ layer and the mobility on the dark $I / V$ curves under small forward bias. Using these insights from the numerical simulation studies, we analyze the dark $I / V$ curves of $\mu c$-Si:H diodes in order to determine the effective defect density and the effective recombination lifetime as a function of the microstructure of the absorber layer, i.e., SC.

\section{EXPERIMENTAL DETAILS}

The $\mu c-\mathrm{Si}: \mathrm{H} \quad p-i-n$ solar cells (glass/ZnO/ $p-i-n / \mathrm{ZnO} / \mathrm{Ag}$ ) examined in this study were deposited by PECVD at $95 \mathrm{MHz}$, at a substrate temperature of $200^{\circ} \mathrm{C}$ and a deposition pressure of 300 mTorr. $^{7}$ The $i$ layers with a thickness of $1 \mu \mathrm{m}$ of the $p-i-n$ diodes were deposited for SC values between $1.5 \%$ (highly crystalline deposition regime) and $7.2 \%$ (amorphous growth regime). The preparation conditions for the doped layers with a thickness of about $20 \mathrm{~nm}$ were kept constant. More details concerning the deposition conditions and material properties, particularly Raman data as a function of SC, are given in Refs. 7, 18, and 19.

\section{EXPERIMENTAL RESULTS}

The effect of the $\mathrm{SC}$ value during the deposition of the absorber layer ( $i$ layer) on the efficiency $\eta$, open-circuit voltage $V_{\mathrm{OC}}$, fill factor $\mathrm{FF}$, and short-circuit current $J_{\mathrm{SC}}$ was previously demonstrated by Vetterl et al. for solar cells with an $n-i-p$ deposition sequence (see Fig. 6 in Ref. 7): the efficiency is small in the highly crystalline deposition regime but increases as the transition region to amorphous growth is approached. Before the transition to predominantly amorphous growth $(\mathrm{SC}=5 \%)$ the efficiency is maximum. A further increase in the SC value leads to a decrease in $\eta$. The same dependence is found for solar cells with a $p-i-n$ deposition sequence. A comparable dependence of the solar cell parameters on the $\mathrm{SC}$ value is also reported by other authors for VHF PECVD, ${ }^{20,21}$ radio-frequency PECVD, ${ }^{3}$ and hotwire CVD. ${ }^{22}$ This fact shows that the behavior is not only characteristic for a particular deposition system or process but is a general property of $\mu c$-Si:H solar cells. No major effect of the texture of the $\mathrm{ZnO}$ substrate on the electronic properties of $\mu c-\mathrm{Si}: \mathrm{H}$ was observed (a correlation between the substrate texture and the electronic properties of $\mu c-\mathrm{Si}: \mathrm{H}$ is proposed by Nasuno et $a l^{2}$ ).

This work focuses on the quantitative analysis of the current-voltage behavior in the dark, particularly for forward bias smaller than $0.1 \mathrm{~V}$, as a function of the $\mathrm{SC}$ value. As will be shown later, these data allow conclusions regarding the effective defect density $N_{d}^{i}$ and the effective recombination

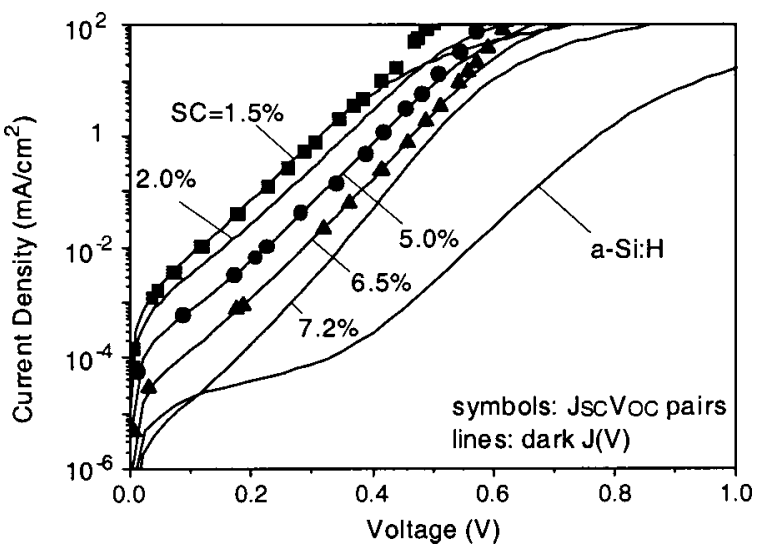

FIG. 1. Dark $I / V$ curves (lines) and $J_{\mathrm{SC}^{-}} V_{\mathrm{SC}}$ pairs (symbols) of $\mu c-\mathrm{Si}: \mathrm{H}$ solar cells with $i$ layers that were deposited with different silane concentrations SC. Also plotted is the dark $I / V$ curve of an amorphous silicon diode with the same $i$ layer thickness $(1 \mu \mathrm{m})$.

lifetime $\tau$ in the center of the $i$ layer. The dark $I / V$ curves are shown in Fig. 1 (lines). In the highly crystalline deposition regime (small SC value), the current density is relatively large. With increasing SC value, the dark current density decreases by several orders of magnitude. The dark saturation current density $J_{0}$ (the current density at the intersection of the extrapolated $I / V$ curve with the ordinate) reaches a value of around $10^{-5} \mathrm{~mA} / \mathrm{cm}^{2}$ for $\mathrm{SC}=5 \%$, where the highest efficiency is achieved within this $\mathrm{SC}$ series $\left(V_{\mathrm{OC}}=520\right.$ $\mathrm{mV}, \mathrm{FF}=70 \%$ ). Increasing the SC value beyond 5\% leads to further decrease in $J_{0}$. Shunt paths by, e.g., cracks in the $i$ layer as a reason for the increase in $J$ with a decreased SC value can be excluded, since these would cause a linear $I / V$ characteristic, in contrast to the typical exponential diode behavior as shown in Fig. 1.

For comparison, the dark $I / V$ curve of an amorphous $\mathrm{Si}$ ( $a$-Si:H) $p-i-n$ diode with the same absorber thickness (1 $\mu \mathrm{m})$ is included. The comparable geometric properties allow direct comparison of the current densities of diodes made of $\mu c-\mathrm{Si}: \mathrm{H}$ and $a$-Si:H. The small $J_{0}$ of the $a$-Si:H diode is mainly caused by the larger mobility gap $E_{\mu}$ and the therefore smaller intrinsic carrier concentration $n_{i}$ due to (see, e.g., Ref. 23)

$$
n_{i}^{2} \propto \exp \left(-E_{\mu} / k T\right) .
$$

Also plotted in Fig. 1 are $J_{\mathrm{SC}^{-}} V_{\mathrm{OC}}$ pairs for diodes that were measured by varying the illumination intensity by more than seven orders of magnitude (for clarity, plotted only for SC $=1.5 \%, 5 \%$, and $6.5 \%$ ). The divergence of the $J_{\mathrm{SC}^{-}} V_{\mathrm{OC}}$ pairs and the dark $I / V$ curves for $J>1 \mathrm{~mA} / \mathrm{cm}^{2}$ is caused by Ohmic series resistance mainly due to the $\mathrm{ZnO}$ layers. For smaller current densities the $J_{\mathrm{SC}}-V_{\mathrm{OC}}$ pairs and the dark $I / V$ curves match well. This shows that the electrostatic potential, the region of the dominant recombination site of injected carriers, and the transport in $\mu c-\mathrm{Si}: \mathrm{H}$ diodes are not strongly affected by the illumination intensity. Hence, the region where most of the recombination of injected electron-hole pairs occurs is similar for operation in the dark and under illumination. ${ }^{24}$ 


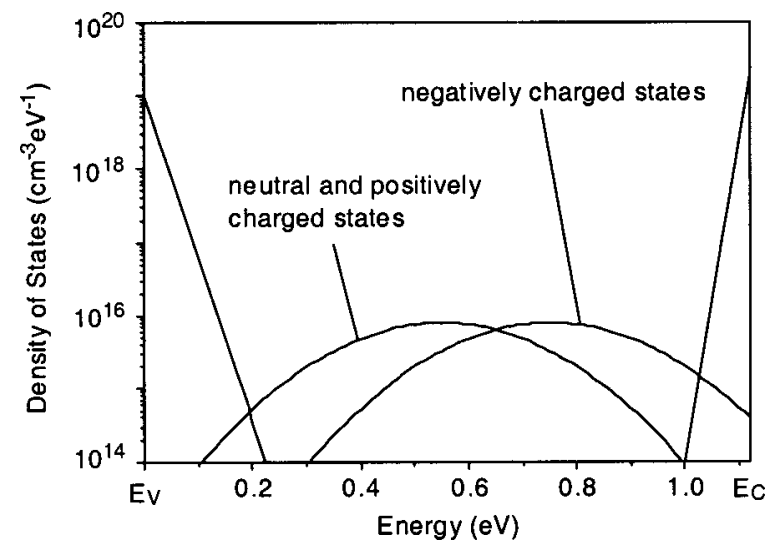

FIG. 2. Density of states as assumed in the numerical model. The peaks of the neutral and positively charged defects are located in the middle of the band gap. A positive correlation energy of $0.2 \mathrm{eV}$ is assumed so that the peak of the negatively charged defects is located above midgap.

\section{NUMERICAL MODEL}

We used a numerical simulation program ${ }^{25}$ which solves the semiconductor equations by the finite-difference method in one dimension. ${ }^{26}$ It was previously used for modeling $a$-Si:H and its alloys, ${ }^{27,28}$ as well as for $\mu c$-Si:H diodes. ${ }^{14,16}$ Recombination and thermal generation are calculated individually at all discrete points from the density of recombination centers, their capture cross section, the position within the band gap, and their Fermi level dependent state of charge. Recombination centers (defects) are assumed to be of positive, neutral, or negative charge if occupied by zero, one, or two electrons, respectively (amphoteric defect statistics $^{29,30}$ for dangling bonds as applied for $\left.a-\mathrm{Si}: \mathrm{H}\right)$. The defect distribution in the bandgap of $\mu c-\mathrm{Si}: \mathrm{H}$ is expected to be fairly broad or even constant with the defect peak close to midgap or slightly shifted to the valence band. ${ }^{31,32}$ Hence, in the numerical simulations the standard deviation of the Gaussian function for the defect distributions is set to 150 $\mathrm{meV}$ (a value also used for amorphous silicon) and the neutral and positive defect peak position is located in the middle of the band gap. Figure 2 shows the density of states as implemented in the numerical simulations. The negative defect peak is elevated by the positive correlation energy of 0.2 $\mathrm{eV}$ above the neutral and positive defect peak as found for $a$-Si:H. ${ }^{33,34}$ The capture cross section of neutral states $\left(\sigma_{N}\right)$ is assumed to be $10^{-16} \mathrm{~cm}^{2}$. The capture cross section for capture of carriers into defects of opposite charge $\sigma_{C}$ (electron (hole) captured by a positively (negatively) charged state) is assumed to be $10^{-15} \mathrm{~cm}^{2}$. The factor of 10 between $\sigma_{C}$ and $\sigma_{N}$ is based on data for $a-\mathrm{Si}: \mathrm{H}$ where Coulombic attraction is made responsible for the increased capture probability into defects of opposite charge. Band details are considered in the simulation program but they have little effect on the analysis presented in the following. The effective densities of states at the band edges as found in crystalline $\mathrm{Si}$ $(c-\mathrm{Si})$ were used. ${ }^{23}$ Throughout this work the hole mobility was assumed to be smaller by a factor of 0.3 as approximately found in $c-\mathrm{Si}^{23}$ and $a-\mathrm{Si}: \mathrm{H}^{27,35}$ where a ratio between 0.1 and 0.3 is used. Published values for the mobilitylifetime product of electrons and holes in $\mu c-\mathrm{Si}: \mathrm{H}$ support

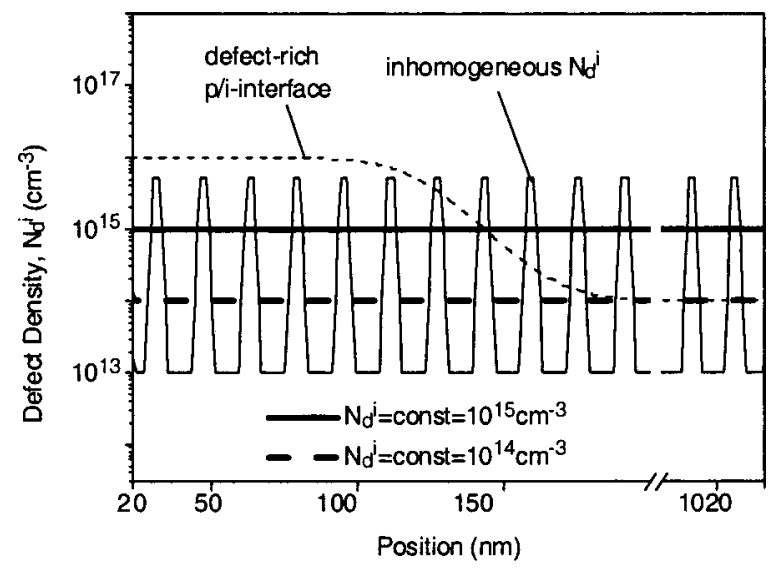

FIG. 3. The spatially resolved profiles in the $i$ layer of different defect densities $N_{d}^{i}(x)$ as implemented in the simulations. The $p$ layer ( $n$ layer) extending from 0 to $20 \mathrm{~nm}(1020$ to $1040 \mathrm{~nm})$ are not shown. Profiles that are constant within the $i$ layer are plotted with bold lines $\left(N_{d}^{i}=10^{14}, 10^{15}\right.$ $\mathrm{cm}^{-3}$ ). The thin dashed line depicts the profile with a defect-rich $p / i$ interface which extends $100 \mathrm{~nm}$ into the $i$ layer $\left(N_{d}^{p / i}=100 \times N_{d}^{i}=10^{16} \mathrm{~cm}^{-3}\right)$. Plotted with the thin solid line is the inhomogeneous $N_{d}^{i}(x)$ with the comblike profile. The corresponding average defect density is $10^{15} \mathrm{~cm}^{-3}$. Note that the abscissa is interrupted between 200 and $980 \mathrm{~nm}$.

this assumption. ${ }^{36}$ From these data it is also concluded that the capture probabilities for electrons and holes in intrinsic $\mu c$-Si:H layers do not differ largely.

\section{DEVICE SIMULATION}

This section deals with the simulation of dark $I / V$ curves of $p-i-n$ diodes for which the above described numerical simulation program is used. In this study the mobility $\mu$ and the defect density in the $i$ layer, $N_{d}^{i}$, are varied. The electron mobility was set to $\mu_{e}=1$ and $1000 \mathrm{~cm}^{2} \mathrm{~V}^{-1} \mathrm{~s}^{-1}$. These values were chosen to cover the range known from $a-\mathrm{Si}: \mathrm{H}^{27,35}$ $\left(\mu_{e}=1-10 \mathrm{~cm}^{2} \mathrm{~V}^{-1} \mathrm{~s}^{-1}\right)$ and $c-\mathrm{Si}^{23}\left(\mu_{e}>100 \mathrm{~cm}^{2} \mathrm{~V}^{-1} \mathrm{~s}^{-1}\right)$. The magnitude of $N_{d}^{i}\left(10^{14}, 10^{15}\right.$, and $\left.10^{16} \mathrm{~cm}^{-3}\right)$ was chosen such that the simulated $J_{0}$ is in the range of the values found experimentally for $\mu c-\mathrm{Si}: \mathrm{H}$ diodes (see Fig. 1). The values for $N_{d}^{i}$ appear small in comparison to the spin densities, which are in the range of $10^{16} \mathrm{~cm}^{-3}$. This is probably due to the overestimation of the capture cross sections in the simulations, which will be discussed later. The general statement, however, is not affected by this discrepancy.

The effect of an inhomogeneous defect density varying spatially on a nanometer scale is also addressed. The motivation for this study is the heterogeneous microstructure of $\mu c-\mathrm{Si}: \mathrm{H}$. The corresponding comblike defect density profile is plotted in Fig. 3 (thin solid line). $N_{d}^{i}=5 \times 10^{15} \mathrm{~cm}^{-3}$ in the narrow peaks with a width of $3 \mathrm{~nm}$. The defect density in the remaining region with a width of $12 \mathrm{~nm}$ is set to $10^{13} \mathrm{~cm}^{-3}$ so that the average defect density in the $i$ layer is $10^{15} \mathrm{~cm}^{-3}$. Also shown in Fig. 3 is the case of a defect-rich region near the $p / i$ interface with a thickness of $100 \mathrm{~nm}$ (thin dashed line). The defect density near the $p / i$ interface is 100 times larger than the defect density in the remaining $i$ layer $\left(N_{d}^{p / i}\right.$ $=10^{16} \mathrm{~cm}^{-3}, N_{d}^{i}=10^{14} \mathrm{~cm}^{-3}$ ). The average defect density in the $i$ layer is consequently $1.1 \times 10^{15} \mathrm{~cm}^{-3}$. For comparison, 


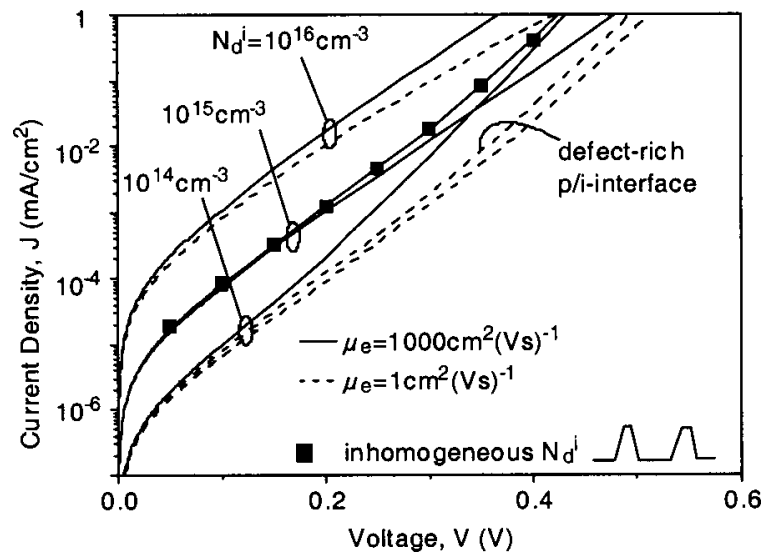

FIG. 4. Simulated dark $I / V$ curves for different constant defect densities $\left(N_{d}^{i}=10^{14}, 10^{15}, 10^{16} \mathrm{~cm}^{-3}\right.$ ), an inhomogeneous defect density (solid squares), and a defect-rich $p / i$ interface. Mobilities of $\mu_{e}=1$ (dashed lines) and $1000 \mathrm{~cm}^{2} \mathrm{~V}^{-1} \mathrm{~s}^{-1}$ (solid lines) were assumed.

Fig. 3 also shows the profiles of constant defect densities with $N_{d}^{i}=10^{14} \mathrm{~cm}^{-3}$ (bold dashed line) and $10^{15} \mathrm{~cm}^{-3}$ (bold solid line).

The simulated dark $I / V$ curves are plotted in Fig. 4. Dark $I / V$ curves can usually be fitted with the function

$$
J(V)=J_{0}\left[\exp \left(\frac{q V}{n k T}\right)-1\right],
$$

with $J_{0}$ called the dark saturation current density, $n$ the ideality factor, $q$ the elementary charge, $k$ Boltzmann's constant, and $T$ the device temperature. The parameters $J_{0}$ and $n$ are used in this work to describe the observed change of the simulated $I / V$ curves upon variation of the input parameters such as $\mu$ and $N_{d}^{i}$. Deviations of a certain $I / V$ curve from a simple exponential law are considered to be due to changes of $n$ with $V(n=n(V))$ while $J_{0}$ is always assumed to be constant. We want to stress, however, that we did not use Eq. (2) but the software described in the previous section to obtain the dark $I / V$ curves discussed next.

The focus of this paper is on the dark $I / V$ curves for small forward bias $(V \leqslant 0.1 \mathrm{~V})$. The most important result of Fig. 4 is that in this bias range $J$ decreases (linearly) with decreasing $N_{d}^{i}$. Additionally, it is of no relevance if the defects are homogeneously distributed $\left(N_{d}^{i}(x)=\right.$ const $)$ or if $N_{d}^{i}(x)$ varies on a nanometer scale. This is shown by a comparison of dark $I / V$ curves for diodes with an average defect density of $10^{15} \mathrm{~cm}^{-3}$. For the diode with the inhomogeneous defect density, $N_{d}^{i}(x)$ has a comblike profile as shown in Fig. 3 (thin solid line). The corresponding dark $I / V$ curve (solid squares) coincides well with the curve for $N_{d}^{i}(x)=$ const $=10^{15} \mathrm{~cm}^{-3}$. Hence, despite the heterogeneous composition of $\mu c-\mathrm{Si}: \mathrm{H}$, which varies within tens of nanometers (for a schematic picture of the microstructure see Fig. 2 in Ref. 7), a simplified approach for the spatial defect distribution can be used because the dark $I / V$ curve of a $\mu c-\mathrm{Si}$ :H diode is not sensitive to the precise defect distribution on a nanometer scale. It is therefore appropriate to regard $N_{d}^{i}$ as the average of the true defect density, which might vary within dimensions small in comparison to the $i$ layer thickness.

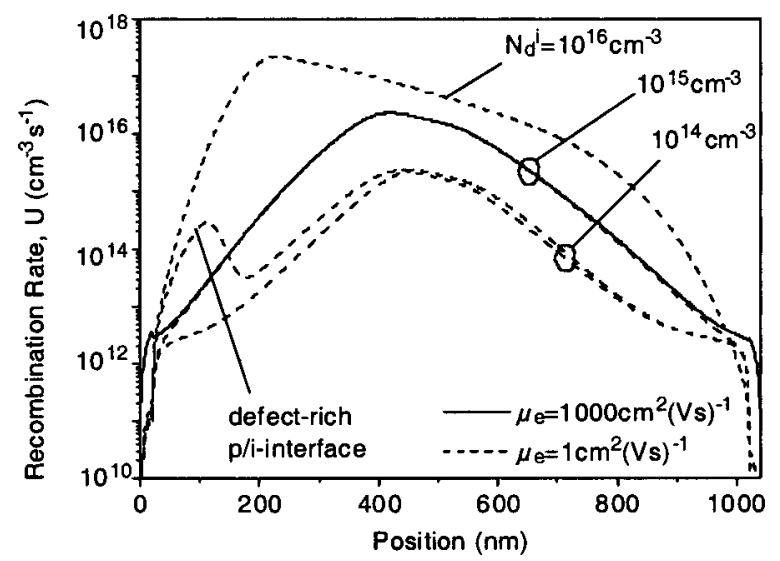

FIG. 5. Net volume recombination rate $U$ in the dark at $0.1 \mathrm{~V}$ forward bias.

Next, the effect of a defect-rich $p / i$ interface on $J_{0}$ is investigated, since a spatial dependence of the defect density on a large scale (in contrast to the variation of $N_{d}^{i}$ within nanometers as investigated above) was detected by, e.g., Svrcek et $a l .{ }^{37}$ As a consequence of the defect-rich $p / i$ interface, $n$ decreases under large forward bias. For $V<0.1 \mathrm{~V}$, however, the dark $I / V$ curve is almost identical with the curve for $N_{d}^{i}=10^{14} \mathrm{~cm}^{-3}$ without a defect-rich $p / i$ interface. Extrapolation of the $I / V$ characteristics for small forward bias shows that $J_{0}$ is in fact not affected by the quality of the $p / i$ interface. This means that $J_{0}$ can be regarded as a measure of the defect density in the center of the $i$ layer, since it is not affected by the properties of the interfaces of the doped layers.

A similar observation is made for the effect of the mobility $\mu$ on the $I / V$ characteristics (compare the solid and dashed lines for one value of $N_{d}^{i}$ in Fig. 4). $J_{0}$ is independent of $\mu$; only $n$ changes. Variation of $\mu$ within the $i$ layer due to, e.g., varying microstructure, will in consequence also have no effect on $J_{0}$.

The simulated spatially resolved recombination rate $U$ is plotted in Fig. 5 in order to explain the impact of a variation in $N_{d}^{i}, N_{d}^{p / i}$, and $\mu$ on the dark current at small forward bias. The $p$ layer is located on the left and the $n$ layer on the right. A voltage of $0.1 \mathrm{~V}$ was chosen since $J$ and $U$ equal zero at 0 $\mathrm{V}$ because of the detailed balance prevailing at thermal equilibrium, so that no conclusions can be drawn from $U(0 \mathrm{~V})$. Integrating $q \times U(x)$ yields the current due to volume recombination (this relationship follows from the continuity equation $^{23}$ ). These integrals change linearly with $N_{d}^{i}$, which explains the earlier mentioned linear dependence of $J_{0}$ on $N_{d}^{i}$. It should be noted here that this relationship is valid only for $J_{0}>10^{-6} \mathrm{~mA} / \mathrm{cm}^{2}$. Below this level, interface and contact recombination become relevant, which changes the dependence of $J_{0}$ on $N_{d}^{i}$.

The characteristic shape of $U(x)$ with the peak in the center of the $i$ layer (note the logarithmic scale) is due to the comparable number of electrons and holes injected from the $n$ layer and the $p$ layer in this region. Close to and in the interface regions, the large difference in the density of holes and electrons reduces $U$ by several orders of magnitude. This is the reason for the insensitivity of the current at small for- 
ward bias to the properties (e.g., defect density, as shown in this work, or mobility gap, as shown in Fig. 5 in Ref. 16) of the doped layers and the region close to them.

The defect-rich $p / i$ interface causes a second peak in $U(x)$ near the $p$ layer. The area underneath the curve, and hence the additional current, is negligible (less than $4 \%$ ) in comparison with the contribution from recombination in the center of the $i$ layer. Only under large forward bias, when the minority carrier density (electrons near and in the $p$ layer) approaches the majority carrier density (holes near and in the $p$ layer), does this region become relevant regarding the dark current density (not shown). The effect of a defect-rich $i / n$ interface is even smaller due to the smaller mobility of holes in comparison to the mobility of electrons.

$U(x)$ for inhomogeneous defect density (comblike profile) is not plotted in Fig. 5 because its large spatial variations by two orders of magnitude would obscure the other recombination rate profiles. It is, however, worth mentioning that $U(x)$ for an inhomogeneous defect density varying on a nanometer scale equals on average $U(x)$ for the case of a constant defect density. In consequence, the diode currents are identical for these two different defect density profiles, which have the same integral defect density as shown in Fig. 4.

The effect of decreasing $\mu_{e}$ from 1000 (solid lines) to 1 $\mathrm{cm}^{2} \mathrm{~V}^{-1} \mathrm{~s}^{-1}$ (dashed lines) on $U(x)$ is also illustrated in Fig. 5 (for clarity, shown only for $N_{d}^{i}=10^{15} \mathrm{~cm}^{-3}$ ). Between 200 and $800 \mathrm{~nm}$ the dashed and solid lines lie on top of each other. $U$ decreases only in the doped layers. This does not significantly affect $J$ due to the extremely small $U$ in these regions.

Summarizing the simulation study, we conclude that $J_{0}$ of $\mu c-\mathrm{Si}: \mathrm{H}$ diodes with an $i$ layer thickness of $1 \mu \mathrm{m}$ is controlled solely by the effective defect density in the central region of the $i$ layer. The diode current is not sensitive to a spatial variation of the defect density if the distance between the recombination sites is much smaller than the thickness of the $i$ layer. $J_{0}$ is also independent of the mobility if $J_{0}$ $>10^{-6} \mathrm{~mA} / \mathrm{cm}^{2}$. Interface related effects such as defect-rich $p / i$ and $i / n$ interfaces do not influence $J_{0}$ but can play an important role under large forward bias. This is also valid for the case of an increased mobility gap of the $i$ layer at the $p / i$ interface due to a large portion of amorphous phase during the initial growth as shown by Zimmer et al. ${ }^{16}$

Based on these simulation results, the dark $I / V$ curves at small voltages of $\mu c-\mathrm{Si}: \mathrm{H} p-i-n$ diodes deposited with different silane concentrations during the deposition of the $i$ layer are analyzed in the next section.

\section{DEVICE ANALYSIS}

In this section the experimental results of Fig. 1 are analyzed to obtain an effective defect density $N_{d}^{i}$ and an effective recombination lifetime $\tau$. By a combination of the experimental data in Fig. 1 and the simulation results in Fig. 4, $N_{d}^{i}$ is evaluated. The result is plotted in Fig. 6 (open triangles). We used SC as the abscissa even though this is only a technological parameter that might deviate for other deposition systems and deposition conditions that achieve films

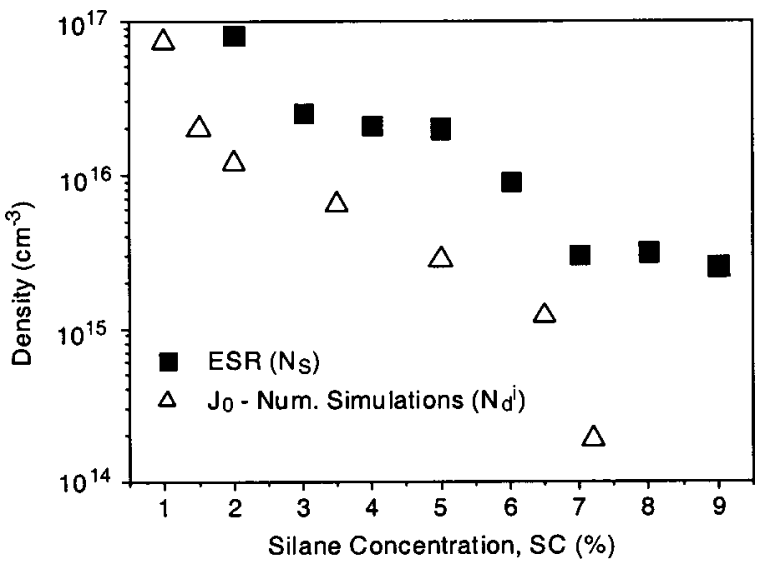

FIG. 6. Defect density in the $i$ layer as a function of the silane concentration $\mathrm{SC}$ as derived from a comparison of experimental data and results from numerical simulations (open triangles). Also plotted are the spin densities (solid squares) of powder samples.

with similar material properties. However, there is no established physical parameter to characterize $\mu c$-Si:H layers.

In the highly crystalline deposition regime (small SC value) $N_{d}^{i}$ is above $10^{16} \mathrm{~cm}^{-3}$. With increasing $\mathrm{SC}$ the transition region to amorphous growth $(\mathrm{SC}=5 \%)$ is approached and $N_{d}^{i}$ decreases by two orders of magnitude. For $\mathrm{SC}>5 \%$, where amorphous growth conditions prevail, photothermal deflection spectroscopy (PDS) gives a hint of an increase of the effective optical band gap. ${ }^{38}$ The mobility gap $E_{\mu}$ strongly affects $n_{i}$ [see Eq. (1)] and, consequently, $J_{0}$, a linear proportionality between $J_{0}$ and $n_{i}$ was found by numerical device simulation, ${ }^{39}$ so that the analysis of the dark $I / V$ curves for which a mobility gap of $1.12 \mathrm{eV}$ was assumed might not be applicable for SC $>5 \%$.

Next, the evaluated defect densities determined by numerical simulations are compared with electron spin resonance (ESR) data. ESR detects the paramagnetic states in a semiconductor. Baia Neto et al. determined the spin densities $N_{S}$ of powder samples of intrinsic layers that were deposited on aluminum foil in the same deposition system as the solar cells investigated here. ${ }^{9}$ These spin densities are also included in Fig. 7 (closed squares). $N_{S}$ decreases with increasing SC value (also reported by Fukawa et $a .^{40}$ ). This is attributed to an improving defect passivation. The difference between $N_{S}(\mathrm{SC})$ and $N_{d}^{i}(\mathrm{SC})$ of about one order of magnitude might occur for several reasons. One reason might be an overestimation of the capture cross section for capture of carriers into states of opposite charge, $\sigma_{C}=10^{-15} \mathrm{~cm}^{-2}$, in the simulation program, since further simulations have

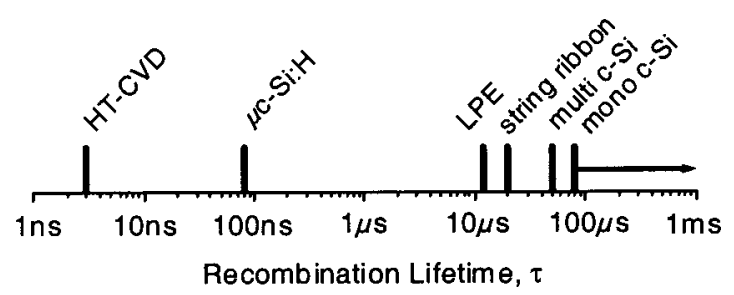

FIG. 7. Comparison of the recombination lifetime in Si fabricated by different technologies. 
shown that $J_{0}$ depends on the product of $\sigma_{C}$ and $N_{d}$. Note that no established data are available regarding the capture cross sections of defects in $\mu c$-Si:H. Also, ESR measurements are relative only to a certain standard, which can cause a deviation from the true spin density by a constant factor.

Provided that $N_{S}(\mathrm{SC})$ represents the true defect density, a comparison of the data from ESR and the dark current analysis can be used to determine the dominant capture cross section. A capture cross section of about $3 \times 10^{-16}$ instead of $10^{-15} \mathrm{~cm}^{-2}$ as used in the simulations would result in similar values for $N_{S}(\mathrm{SC})$ and $N_{d}^{i}(\mathrm{SC})$ for $\mathrm{SC} \leqslant 5 \%$.

Due to the uncertainty in the capture cross section and the absolute magnitude of the reported spin densities, the gradients of $N_{d}^{i}(\mathrm{SC})$ and $N_{S}(\mathrm{SC})$ will be discussed rather than the absolute values. For $\mathrm{SC} \leqslant 3 \% N_{d}^{i}(\mathrm{SC})$ and $N_{S}(\mathrm{SC})$ change similarly with SC. For $3 \%<\mathrm{SC} \leqslant 5 \% N_{S}(\mathrm{SC})$ is almost constant while $N_{d}^{i}(\mathrm{SC})$ varies by a factor of approximately 5. For SC $>5 \%$ the gradients of $N_{d}^{i}(\mathrm{SC})$ and $N_{S}(\mathrm{SC})$ deviate considerably. For this $\mathrm{SC}$ range, the previously mentioned uncertainty in $n_{i}$ caused by the large amorphous volume fraction is the likely reason for the different dependence of $N_{d}^{i}(\mathrm{SC})$ and $N_{S}(\mathrm{SC})$ on $\mathrm{SC}$. The general question also arises as to how far the electronic properties (spin densities) determined on chemically treated powder samples of layers deposited on aluminum foil can be compared with the properties of $i$ layers in completed diodes that were deposited on a (doped) $\mu c-\mathrm{Si}: \mathrm{H}$ layer. Additionally, ESR detects only paramagnetic states (dangling bonds occupied by a single electron, hence neutral states). A shift of the Fermi level could consequently affect $N_{S}$. Compensative doping experiments were carried out as reported in Refs. 7 and 41, and it was proved that the conductivity is minimum without additional doping, so we conclude that the Fermi level of the intrinsic layers is close to the midgap (neglecting the possibly different effective densities of states at the band edges). However, the exposure to air and chemical treatment of the ESR powder samples might shift the Fermi level near the surface due to, e.g., surface reactions.

As an alternative to ESR, the absorption coefficient at $0.8-0.9 \mathrm{eV}$ as determined by PDS is often used to determine the density of the defect states. However, no correlation exists between the absorption coefficient and the spin density for our samples. ${ }^{42}$ Stress and the uncertainty in the amorphous volume fraction might be responsible for the difficult quantitative evaluation of the optical properties. ${ }^{43}$

The recombination lifetime $\tau$ can be calculated by assuming a single defect level in the middle of the band gap with a density of $N_{t}$ instead of the broad defect distribution with density $N_{d}^{i}$. The real defect density $N_{d}^{i}$ is, hence, condensed on a single level. According to the Shockley-ReadHall statistics (see also, e.g., Ref. 44, pp. 295, 438), the recombination lifetime for holes and electrons $\tau$ in the center of the $i$ layer is then

$$
\tau=2 /\left(v_{\mathrm{th}} \sigma N_{t}\right),
$$

where $v_{\text {th }}$ is the thermal velocity $\left(10^{7} \mathrm{~cm} / \mathrm{s}\right)$ and $\sigma$ is the dominant capture cross section of the defect. In the numerical simulations a single defect level was realized by reducing the standard deviation of the Gaussian function for the defect distributions from 150 to $1.5 \mathrm{meV}$. With this adjustment the defect density, now termed $N_{t}$, was again varied to find a match with the experimental dark $I / V$ curves of Fig. 1. Determining $N_{t}$ in this manner, one finds a value that is only about a factor of 3 smaller than the previously obtained value for $N_{d}^{i}$. Using this new value for $N_{t}$ and setting $\sigma=\sigma_{C}$ $=10^{-15} \mathrm{~cm}^{2}$, since the capture into charged states dominates, Eq. (3) yields $\tau$. For SC $=5 \%$, where the highest solar cell efficiencies are achieved, $\tau$ was found to equal $80 \mathrm{~ns}$. In the highly crystalline deposition regime $\tau$ is smaller than $1 \mathrm{~ns}$.

The value for $\tau$ of $80 \mathrm{~ns}$ derived here allows a comparison with other Si technologies as shown in Fig. 7. Plotted are recombination lifetimes reported for high temperature CVD (HT-CVD) Si on glass, ${ }^{45}$ liquid phase epitaxial (LPE) grown $\mathrm{Si}$ on monocrystalline $\mathrm{Si},{ }^{46}$ string ribbon grown $\mathrm{Si}^{47}$ multi-${ }^{48}$ and monocrystalline $\mathrm{Si}^{49,50}$ The graph shows that $\tau$ in crystalline $\mathrm{Si}$ as used for solar cell applications is three to four orders of magnitude larger than in $\mu c-\mathrm{Si}: \mathrm{H}$ absorbers for thin-film solar cells deposited by VHF PECVD.

\section{DISCUSSION}

In the following, several aspects of the analysis of the dark current to evaluate $N_{d}^{i}$ and $\tau$ are addressed. The terms "effective" defect density and "effective" recombination lifetime were introduced since we used a one-dimensional model. Microcrystalline silicon is, however, a heterogeneous material so that a three-dimensional model would be required to model the transport and recombination precisely. But little is known about, e.g., the location of the states that are detected by ESR (according to various reports the defects are located at the grain boundaries ${ }^{51-53}$ ), the electronic properties of the coherent domains and their boundaries, the grain boundaries, and the internal surfaces due to voids within the layer. While more insight into these properties is necessary to achieve a detailed microscopic picture, the simulations with an inhomogeneous defect density (solid squares in Fig. 4) proved that the one-dimensional model gives a reasonably good picture of $\mu c-\mathrm{Si}: \mathrm{H}$ despite its heterogeneous microstructure.

The derivation of Eq. (3) includes the assumption that the excess charge carrier concentrations are much larger than the equilibrium carrier concentrations. For a $p-i-n$ diode this assumption is valid only for the center of the $i$ layer. It is not valid in and close to the doped layers due to the in-diffusion of charge carriers from the doped layers. In consequence, $\tau$ derived in this work is the lifetime in the middle of the $i$ layer of a $p-i-n$ diode. It corresponds to the lifetime in an individual $i$ layer as used for, e.g., measurement of the steady-state photoconductivity, steady-state photocarrier grating, or time of flight (neglecting the effect of the asymmetric effective density of states at the band edges).

The simulations showed that the change in the dark current with different SC can be explained by a change of $\tau$ in the center of the $i$ layer. A qualitative analysis was carried out by assuming a broad Gaussian defect distribution with the peak of the neutral and positive states in the middle of the band gap. The assumption of a broad defect distribution 
with a peak located close to midgap is based on ESR studies. ${ }^{31,32}$ These studies, however, give only a preliminary picture of the defect distribution in $\mu c-\mathrm{Si}: \mathrm{H}$, since Poruba et al. propose that the peak of the singly occupied dangling bond states is located $0.3-0.35 \mathrm{eV}$ above the valence band. ${ }^{54}$ Additionally, possible changes of the defect peak level with a variation in SC have not been investigated in detail. Hence, it cannot be excluded that the defect peak varies with SC. The defect peak location affects the recombination rate $U$. In consequence, the dark current also varies with the defect peak level. If the defect peak level varied with the SC value, the derivation of $N_{d}^{i}$ from the dark current as a function of SC would be erroneous. The derivation of $\tau$ from the dark current, however, is not affected by the uncertainty in the defect peak level.

Temperature-dependent measurements in the range from 250 to $350 \mathrm{~K}$ were carried out in order to determine the activation energy $E_{a}$ defined as

$$
J_{0}(T)=J_{00} \exp \left(-\frac{E_{a}}{k T}\right) .
$$

The result is comparable with the measurements by Meier et al. ${ }^{55} E_{a}$ increases from about $0.5 \mathrm{eV}$ in the highly crystalline deposition regime $(\mathrm{SC}=2 \%)$ to about $0.7 \mathrm{eV}$ where the highest efficiency is achieved $(\mathrm{SC}=5 \%)$. For higher $\mathrm{SC}$ values, $E_{a}$ approaches $1 \mathrm{eV}$. Provided that $E_{\mu}$ is constant $(\mathrm{SC} \leqslant 5 \%)$ the increase of $E_{a}$ with increasing SC indicates that the operation conditions of the diode shift from high level injection (recombination takes mainly place in the center of the $i$ layer) to low level injection conditions (recombination occurs mainly in and close to the doped layers). ${ }^{56,57}$ This supports the results shown in Fig. 6. However, we want to stress that determining $E_{a}$ is difficult due to the superposition of parasitic resistance particularly at low and high temperatures. For $\mathrm{SC}>5 \%$ the increase in $E_{a}$ is probably caused by the increasing $E_{\mu}$ as indicated by PDS. ${ }^{38}$

There are no recent reports on $\tau$ in $\mu c$-Si:H as implemented in devices. Usually, $\mu$ or $\mu \tau$ is determined. ${ }^{58-60}$ To our knowledge, there are no reports in which both were determined on the same sample which would allow the derivation of $\tau$. In order to compare $\tau$ determined in this work with material parameters determined by others we verify our data as follows. Assuming a mobility of $3 \mathrm{~cm}^{2} / \mathrm{V} \mathrm{s}$ and with $\tau$ $=80 \mathrm{~ns}$ as determined in this work for material that results in the highest solar cell efficiency, the diffusion length $L$ in the absorber layer ${ }^{23}$

$$
L=\sqrt{(k T / q) \mu \tau}
$$

would be around $0.9 \mu \mathrm{m}$. This is in the range of $0.2-1.5 \mu \mathrm{m}$ covered by measurements of other groups. ${ }^{10,36,61,62}$ Exceptions are the reports by Yamamoto et al. in which $L$ exceeds $7 \mu \mathrm{m} .{ }^{63,64}$ This would correspond to a 100 times larger $\mu \tau$ in comparison to the data from other groups.

\section{CONCLUSIONS}

The general effect of changes in the magnitude of the defect density and the mobility in the intrinsic layer on the dark characteristics of thin diodes with $p-i-n$ structure was demonstrated using numerical device simulations. It was shown that the defect density and the recombination lifetime in the intrinsic layer of microcrystalline silicon solar cells can be deduced from the dark diode characteristics at small voltages, since the device is then fully controlled by recombination in the absorber layer. The derived defect density has to be regarded as an effective parameter since it is of no relevance for the diode current if the recombination sites vary on a nanometer scale. The diode current is also not affected by the material properties of the $i$ layer close to the doped layers. Under large forward bias, however, these regions become important. Comparison with experimental data shows that the effective defect density of microcrystalline silicon decreases as the growth conditions shift from the highly crystalline deposition conditions (small SC value) toward the transition region to amorphous growth (medium SC value), where the highest solar cell efficiencies are obtained. The spin densities of powder samples show a similar quantitative trend in the highly crystalline deposition region, but a considerable deviation is found close to the transition region to amorphous growth. Near the transition region the amorphous volume fraction is probably not negligible but not precisely known. This causes an uncertainty in the intrinsic carrier concentration which can lead to an erroneous defect density by a misinterpretation of the dark $I / V$ curves. Combining ESR data and the analysis of the dark current density, the capture cross section of the dominant recombination center is estimated to be $3 \times 10^{-16} \mathrm{~cm}^{-2}$. For state-of-the-art PECVD $\mu c-\mathrm{Si}: \mathrm{H}$ solar cells, the effective recombination lifetime in the absorber is about $80 \mathrm{~ns}$.

\section{ACKNOWLEDGMENTS}

The authors are grateful for the contributions of and helpful discussions with A. Lambertz, O. Vetterl, L. Houben, F. Finger, R. Carius, and B. Rech.

${ }^{1}$ S. Klein, F. Finger, R. Carius, O. Kluth, L. Baia Neto, H. Wagner, and M. Stutzmann, in Proceedings of the 17th European Photovoltaic Solar Energy Conference and Exhibition (WIP Renewable Energies, München, Germany, 2002), pp. 2965-2968.

${ }^{2}$ Y. Nasuno, M. Kondo, and A. Matsuda, Jpn. J. Appl. Phys., Part 2 40, L303 (2001)

${ }^{3}$ T. Repmann, W. Appenzeller, T. Roschek, B. Rech, O. Kluth, and H. Wagner, in Proceedings of the 17th European Photovoltaic Solar Energy Conference and Exhibition (Ref. 1), pp. 2836-2841.

${ }^{4}$ K. Yamamoto, M. Yoshimi, Y. Tawada, S. Fukuda, T. Sawada, T. Meguro, H. Takata, T. Suezaki, Y. Koi, K. Hayashi, T. Suzuki, M. Ichikawa, and A. Nakajima, Sol. Energy Mater. Sol. Cells 74, 449 (2002).

${ }^{5}$ J. Meier, S. Dubail, S. Golay, U. Kroll, S. Fay, E. Vallat-Sauvain, L. Feitknecht, J. Dubail, and A. Shah, Sol. Energy Mater. Sol. Cells 74, 457 (2002).

${ }^{6}$ M. Luysberg, C. Scholten, L. Houben, O. Vetterl, R. Carius, and F. Finger, Mater. Res. Soc. Symp. Proc. 664, A15.2 (2001).

${ }^{7}$ O. Vetterl, F. Finger, R. Carius, P. Hapke, L. Houben, O. Kluth, A. Lambertz, A. Mück, B. Rech, and H. Wagner, Sol. Energy Mater. Sol. Cells 62, 97 (2000).

${ }^{8}$ J. Bailat, E. Vallat-Sauvain, L. Feitknecht, C. Droz, and A. Shah, J. NonCryst. Solids 299-302, 1219 (2002).

${ }^{9}$ A. L. Baia Neto, A. Lambertz, R. Carius, and F. Finger, Phys. Status Solidi A 186, R4 (2001).

${ }^{10}$ N. Wyrsch, C. Droz, L. Feitknecht, P. Torres, E. Sallat-Sauvain, J. Bailat, and A. Shah, J. Non-Cryst. Solids 299-302, 390 (2002).

${ }^{11}$ T. Fujisaki, A. Yamada, and M. Konagai, Sol. Energy Mater. Sol. Cells 74, 331 (2002). 
${ }^{12}$ K. Taretto, U. Rau, and J. H. Werner, Solid State Phenom. 80-81, 311 (2001).

${ }^{13}$ H. Takakura and Y. Hamakawa, Sol. Energy Mater. Sol. Cells 74, 479 (2002).

${ }^{14}$ J. Zimmer, H. Stiebig, P. Hapke, and H. Wagner, in Proceedings of the 2nd World Conference and Exhibition on Photovoltaic Solar Energy Conversion (James \& James, London, 1998), pp. 944-947.

${ }^{15}$ A. Fantoni, M. Vieira, and H. Wagner, Solid-State Electron 43, 1709 (1999).

${ }^{16}$ J. Zimmer, H. Stiebig, and H. Wagner, Mater. Res. Soc. Symp. Proc., 507, 377 (1998).

${ }^{17}$ L. Houben, M. Luysberg, P. Hapke, R. Carius, F. Finger, and H. Wagner, Philos. Mag. A 6, 1447 (1997).

${ }^{18}$ F. Finger, S. Klein, T. Dylla, A. L. Baia Neto, O. Vetterl, and R. Carius, Mater. Res. Soc. Symp. Proc. (to be published).

${ }^{19}$ O. Vetterl, A. Groß, T. Jana, S. Ray, A. Lambertz, R. Carius, and F. Finger, J. Non-Cryst. Solids 299-302, 772 (2002).

${ }^{20}$ J. Meier, E. Sallat-Sauvain, S. Dubail, U. Kroll, J. Dubail, S. Golay, L. Feitknecht, P. Torres, S. Fay, D. Fischer, and A. Shah, Sol. Energy Mater. Sol. Cells 66, 73 (2001).

${ }^{21}$ T. Matsui, M. Tsukiji, H. Saika, T. Toyama, and H. Okamoto, in Technical Digest of the 12th International Photovoltaic Science and Engineering Conference (Kyung Hee Information Printing, Seoul, Korea, 2001), pp. $355-356$

${ }^{22}$ S. Klein, J. Wolff, F. Finger, R. Carius, H. Wagner, and M. Stutzmann, Jpn. J. Appl. Phys., Part 2 41, L10 (2002).

${ }^{23}$ S. M. Sze, Physics of Semiconductor Devices (Wiley, New York, 1981).

${ }^{24} \mathrm{M}$. Wolf and H. Rauschenbach, Adv. Energy Conversion 3, 455 (1963).

${ }^{25}$ H. Stiebig and M. Böhm, J. Non-Cryst. Solids 164-166, 785 (1993).

${ }^{26}$ M. Kurata, Numerical Analysis for Semiconductor Devices (Lexington Books, Lexington, MA, 1982)

${ }^{27}$ J. Zimmer, H. Stiebig, and H. Wagner, J. Appl. Phys. 84, 611 (1998).

${ }^{28}$ J. Zimmer, D. Knipp, H. Stiebig, and H. Wagner, IEEE Trans. Electron Devices 46, 884 (1999).

${ }^{29}$ N. F. Mott and E. A. Davis, Electronic Processes in Non-Crystalline Materials (Clarendon, Oxford, 1979).

${ }^{30}$ R. A. Street and K. Winer, Phys. Rev. B 40, 6236 (1989).

${ }^{31}$ J. Müller, Ph.D. thesis, University of Aachen, 1998.

${ }^{32}$ P. Kanschat, H. Mell, K. Lips, and W. Fuhs, Mater. Res. Soc. Symp. Proc. 609, A27.3 (2000).

${ }^{33}$ M. Stutzmann and W. B. Jackson, Solid State Commun. 62, 153 (1987).

${ }^{34}$ G. Schumm and G. H. Bauer, J. Non-Cryst. Solids 137-138, 315 (1991)

${ }^{35}$ R. A. Street, Hydrogenated Amorphous Silicon (Cambridge University Press, Cambridge, England, 1991)

${ }^{36}$ M. Goerlitzer, P. Torres, N. Beck, N. Wyrsch, H. Keppner, J. Pohl, and A. Shah, J. Non-Cryst. Solids 227-230, 996 (1998).

${ }^{37}$ V. Svrcek, I. Pelant, J. Kocka, J. Stuchlik, A. Fejfar, and J. Kocka, Thin Solid Films 383, 271 (2001).

${ }^{38}$ F. Finger, O. Vetterl, R. Carius, A. Lambertz, C. Scholten, L. Houben, and M. Luysberg, in Proceedings of the 11th International School on Condensed Matter Physics, Varna, Bulgaria, edited by J. M. Marshall, A. G. Petrov, A. Vavrek, D. Nesheva, D. Dimova-Malinovska, and J. M. Maud (Bookcraft, Bath, England, 2001), p. 26.
${ }^{39}$ T. Brammer, Ph.D. thesis, University of Düsseldorf, Germany, 2002, http://www.ulb.uni-duesseldorf.de/diss/mathnat/2002/brammer.html (in German).

${ }^{40}$ M. Fukawa, S. Suzuki, L. Guo, M. Kondo, and A. Matsuda, Sol. Energy Mater. Sol. Cells 66, 217 (2001).

${ }^{41}$ T. Dylla, R. Carius, and F. Finger, Mater. Res. Soc. Symp. Proc. 715, 333 (2002).

${ }^{42}$ O. Vetterl, A. Groß, T. Jana, S. Ray, A. Lambertz, R. Carius, and F. Finger, J. Non-Cryst. Solids 299-302, 772 (2002).

${ }^{43}$ K. H. Jun, R. Carius, and H. Stiebig, Phys. Rev. B 66, 115301 (2002).

${ }^{44}$ C. T. Sah, Fundamentals of Solid-State Electronics (World Scientific, Singapore, 1991)

${ }^{45}$ R. Brendel, R. B. Bergmann, P. Lölgen, M. Wolf, and J. H. Werner, Appl. Phys. Lett. 70, 390 (1997).

${ }^{46}$ K. J. Catchpole, K. J. Weber, A. B. Sproul, and A. W. Blakers, in Proceedings of the 2nd World Conference and Exhibition on Photovoltaic Solar Energy Conversion (Ref. 14), pp. 1336-1339.

${ }^{47}$ A. Rohatgi, A. Ebong, V. Yelundur, and A. Ristow, Prog. Photovoltaics 8, 515 (2000).

${ }^{48}$ A. Cuevas, M. Stocks, S. Armand, M. Stuckings, A. W. Blakers, and F. Ferrazza, Appl. Phys. Lett. 70, 1017 (1997).

${ }^{49}$ S. W. Glunz, S. Rein, W. Warta, J. Knobloch, and W. Wettling, Sol. Energy Mater. Sol. Cells 65, 219 (2001).

${ }^{50}$ A. Cuevas, Sol. Energy Mater. Sol. Cells 57, 277 (1999).

${ }^{51}$ F. Finger, J. Müller, C. Malten, and H. Wagner, Philos. Mag. B 77, 805 (1998).

${ }^{52}$ W. Fuhs, P. Kanschat, and K. Lips, J. Vac. Sci. Technol. B 18, 1792 (2000).

${ }^{53}$ M. Kondo, S. Yamasaki, and A. Matsuda, J. Non-Cryst. Solids 266-269, 544 (2000).

${ }^{54}$ A. Poruba, M. Vanecek, J. Meier, and A. Shah, J. Non-Cryst. Solids 299302, 536 (2002).

${ }^{55}$ J. Meier, H. Keppner, S. Dubail, U. Kroll, P. Torres, P. Pernet, Y. Ziegler, J. A. Anna Selvan, J. Cuperus, D. Fischer, and A. Shah, Mater. Res. Soc. Symp. Proc. 507, 139 (1998).

${ }^{56} \mathrm{~T}$. Brammer and H. Stiebig (unpublished).

${ }^{57}$ M. S. Bennett and R. R. Arya, Sol. Cells 18, 289 (1986).

${ }^{58}$ G. Juska, K. Arlauskas, and M. Viliunas, Phys. Rev. Lett. 84, 4946 (2000).

${ }^{59}$ T. Sameshima, K. Saitoh, N. Aoyama, M. Tanda, M. Kondo, A. Matsuda, and S. Higashi, Sol. Energy Mater. Sol. Cells 66, 389 (2001).

${ }^{60}$ K. Nakahata, A. Miida, T. Kamiya, C. M. Fortmann, and I. Shimizu, Thin Solid Films 337, 45 (1999).

${ }^{61}$ K. Nakahata, T. Kamiya, C. M. Fortmann, I. Shimizu, H. Stuchlikova, A. Fejfar, and J. Kocka, J. Non-Cryst. Solids 266-269, 341 (2000).

${ }^{62}$ V. Svrcek, I. Pelant, J. Kocka, P. Foitik, B. Rezek, H. Stuchlikova, A. Fejfar, J. Stuchlik, and A. Poruba, J. Appl. Phys. 89, 1800 (2001).

${ }^{63}$ K. Yamamoto, M. Yoshimi, T. Suzuki, Y. Tawada, Y. Okamoto, and A. Nakajima, in Proceedings of the 2nd World Conference and Exhibition on Photovoltaic Solar Energy Conversion (Ref. 14), pp. 1284-1289.

${ }^{64} \mathrm{~K}$. Yamamoto, M. Yoshimi, Y. Tawada, Y. Okamoto, and A. Nakajima, J. Non-Cryst. Solids 266-269, 1082 (2000). 